\title{
S6 Figure.
}

\section{LIN9 SM}

\section{3' boundaries}

28S rDNA AAAGCGACGTCGCTATGAACGCTTGGCCGCCACAAGCCAGTTATCCCTGTGGTAACTTTTCTGACACCTCTTGCTACAAACTCTGTAAAAATCAAAAGGATCGATAGGCCGCGCTTTCAC

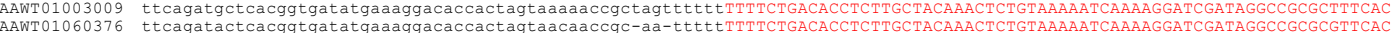
AAWT01078088 ttcagatgctcgcggtgatatgaatggacaccactagtaaaaccgctag-tttttTTTTCTGACACCTCTTGCTTCAAACTCTGAAGACATCAAAAAGGATCAA

AAWT01077286 ttcagatgctcacggtgatatgaa

AAWT01088271 ctcagatgctcacggtgatatgaaaagacaacactagtaaaaccgctag-tttatTTTTCTGACACTTCTTGCTACAAACTCTGTAAAAATCAAAAGGGATCGATAGGCCGCGCTTTCAC

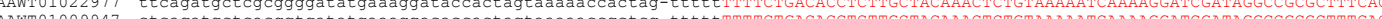
AAWT01030948 ttcagacgctcacggtgacaagaaagaacaccactagtaaaaccactagtag---TTTCTGACACTCTTGCTTCAACTCTGAAGACACAAAAAGGATCGAAGGCCGCGCTICAC

\section{5' boundaries}

28S rDNA AAAGCGACGTCGCTATGAACGCTTGGCCGCCACAAGCCAGTTATCCCTGTGGTAACTTTTCTGACACCTCTTGCTACAAACTCTGTAAAAATCAAAAGGATCGATAGGCCGCGCTTTCAC AAWT01008734 AAAGCGACGTCGCTATGAACGCTTGGCCGCCACAAGCCAGTTATCCCTGCGGTAACattgtggaactcataagacaagtactaaagaagaattagaaaattagaagaaaaattgaad AAWT01077338 - AAWT01044953 AAAGCGACGTCGCTATGAACGCTTGGCCGCCACAAGCCAGTTATCCCTGTGGTAACattgtggaactcataagacaagtactaaaagagaattagaaaattagaagaaaaattgaa AAWTO1081629 AAA-CGACAT--C-ATGAACGCTIGGCCGCAACAATCCAGTTATCCCTGCGGTAACattgtggaactcataagacaagtactaaaagaagaattagaaaattagaagaaaaa ttgaa AAWT01008734 AAA-CGACAT-C-A-ATGAACGCTTGGCCGCAACAATCCAGTTATCCCTGCGGTAACattgtggaactcataagacaagtactaaagaagaattagaaaattagaagaaaaattgaaa AAWT01008741 AAA-CGACAT--C-ATGAACGCTTGGCCACAACAATCCAGTIATCCCTGCGGTAACattgtggaactcataagacaagtactaaagaagaattagaaaattagaagaaaaattgaa AAWT01044928 AAA-CGACA--AC-ATGAACGCTTGGCCGCAACAATCCAGTTATCCCTGCGGTAACattgtggaactcataagacaagtactaaagaagaattagaaaaattagaagaaaaattgaaa

\section{LIN15_SM}

\section{3' boundaries}

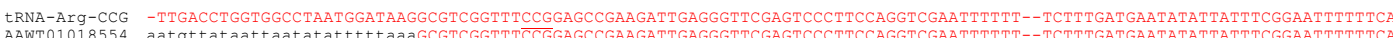
AAWT01076251 aatgttataattaatatattttaaaGCGTCGGTTTCCGGAGCCGAAGATTGAGGGTTCGAGTCCCTTCCAGGTCGAATTTTTTT--TCTTTGATGAATATATTATTTTCGGAATTTTTTTCA

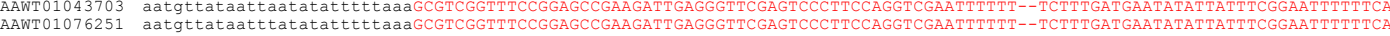

tRNA-Arg-CCT TAAGCCCGCGTGGCCTAATGGATAAGGCGTCTGCCTCCTAAGCAGAAGATTTGCGGGTTCGAGTCCCGCCGTGGGTAAATTTTAT--TCTCAACTAGAAATTTTAAAAATTTAGAAAATTGA

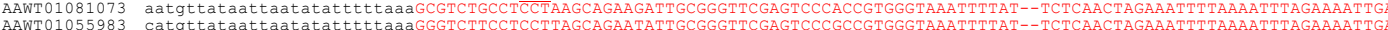
AAWT01009870 aatgttataat AAWT01027286 aatgttataattaatatattttaaaGCGTCTGCCTCCTAAGCAGAAGATTGCGGGTTCGAGTCCCGCCGTGGGTAAATTTTTAT--TCTCAACTAGAAATTT TAAAAATTTAGAAAATTGA RNA-Arg-TCG TAAGGCCCTGTGGCCCAATGGATAAGGCGTCTGACTTCGAATCAGAAGATTTGCAGGTTCGAGTCCTGCCAGGGTCGTATTTTTTTGTCATTCTTGTTAATTCTAATTTTTAGGCCCTGCA AAWT0 1076691 aatgttatgattaatatatttttaaaGCGTCTGACTTCGAATCAGAAGATTGCAGGTTCGAGTCCTGCCAGGGTCGTATTTTTTTTGTCATTCTTGTTAATTCTAATTTTTAGGCCCTGCA

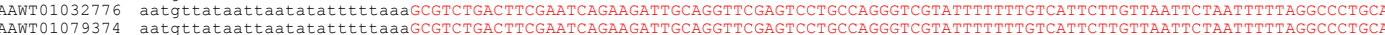
tRNA-Arg-TCT AGAGTCTCAGTGGCGCAATGGATAGCGCGTCGGACTTCTAATCCGGAGGTTGTGGGTTCGATCCCACCTGAGATGCATTTTTACAGAATTTTGAGTGAGAAAATTCTGTTTTGTTTTCA AAWT01004690 aatgttataattaatatattttaaaGCGTCGGACTTCTAATCCGGAGGTTGTGGGTTCGAGTCCCACCTGAGATGCATTTTTACAGAATTTTGAGTGAGAAAAATTCTGTTTTGTTTTCA

\section{5 ' boundaries}

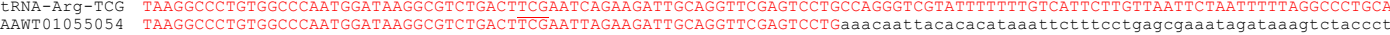

\section{LIN24 SM}

\section{3' boundaries}

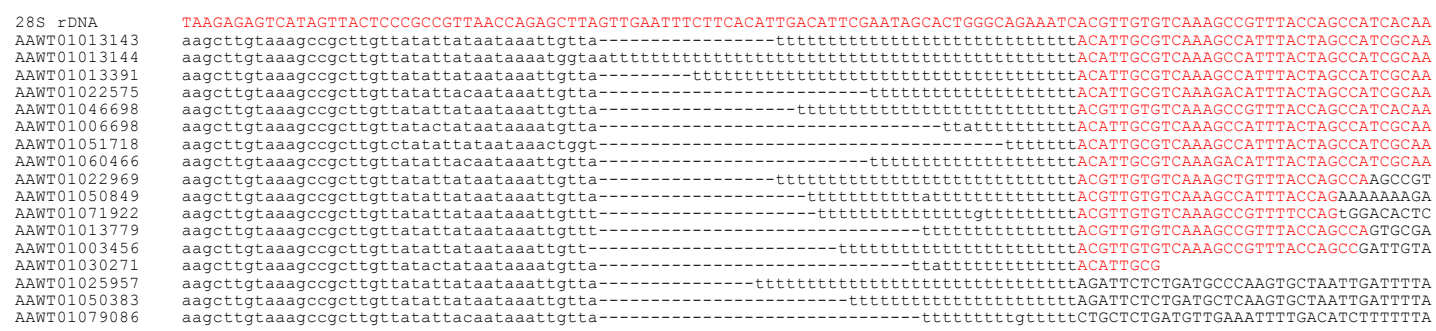

\section{LIN25_SM}

\section{3' boundaries}

$\begin{array}{ll}\text { tRNA-Glu-TTC } & \text { gatgaatatttggtaataatattataattaactaaTCCCTGATGGTCTAGCGGTTAGGATTCCTGGTTTTCACCCAGGCGGCCCGGGTTCGACTCCCGGTCAGGGA } \\ \text { AAWT01038405 } & \text { gtaaaattgacaatt }\end{array}$ 


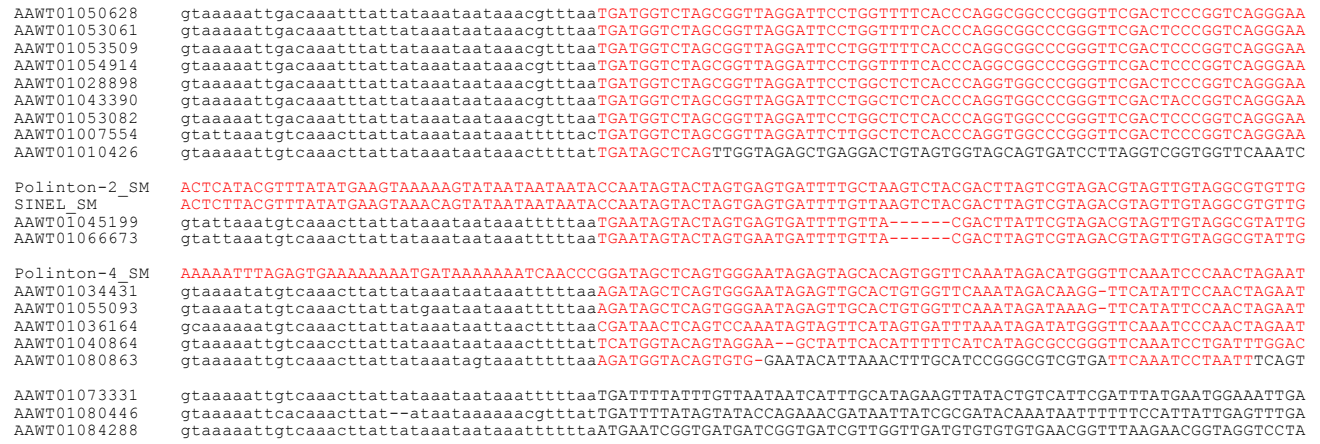

\section{LIN26_SM}

\section{3' boundaries}

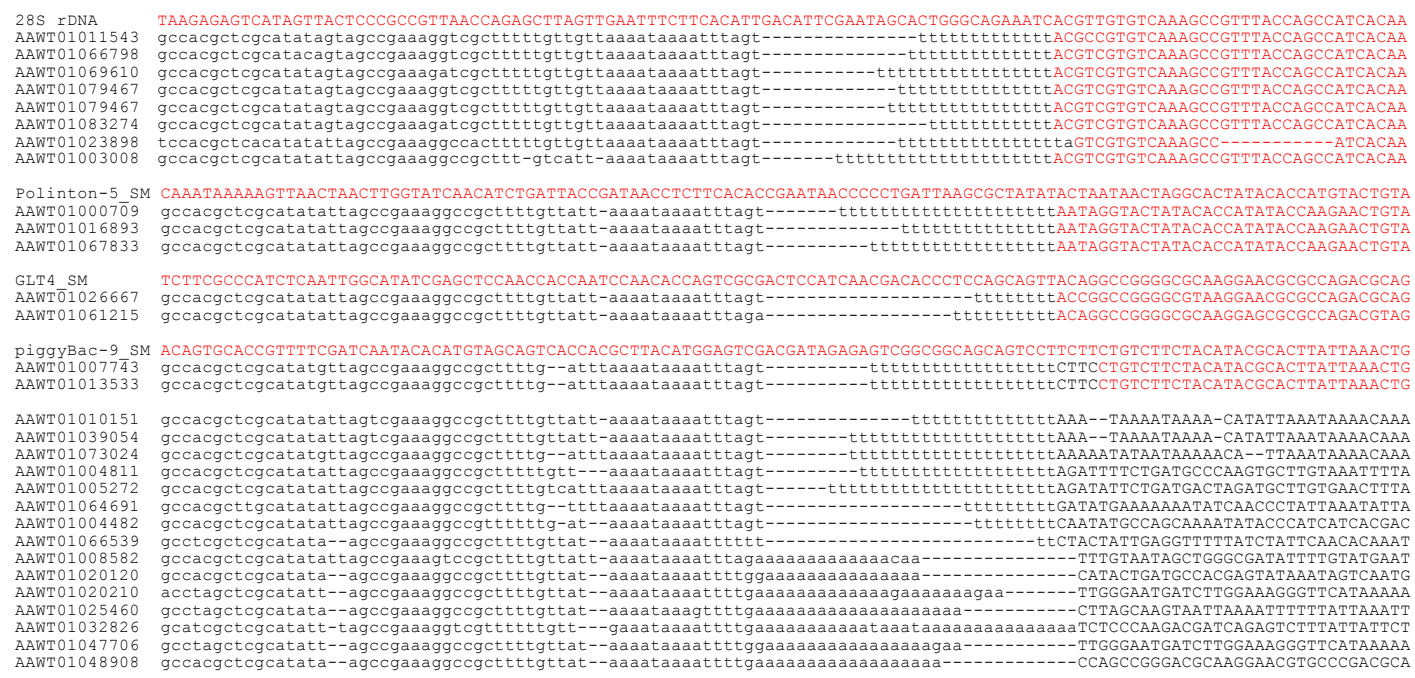

\section{NeSL-1_TV}

\section{3' boundaries}

\begin{tabular}{|c|c|}
\hline $\begin{array}{l}\text { tRNA-Pro-TGG } \\
\text { DS113626 } \\
\text { DS114997 } \\
\text { DS113485 } \\
\text { DS115424 } \\
\text { DS133744 }\end{array}$ & 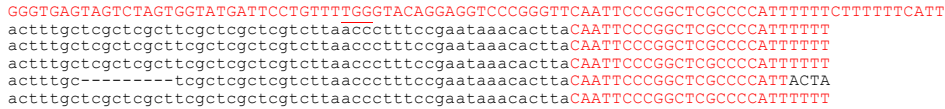 \\
\hline
\end{tabular}

\section{5' boundaries}

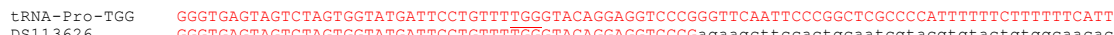
DS113485 GGGTGAGTAGTCTAGTGGTATATTCCTGTTTTGGGACAGGAGGTCCCGagaagcttccactgcatcgtacgtgtactgtggcaacacagctataacagacagttcacgccaaccge DS113187 GGGTGAGGAGTCTAGTGGTATGATTCCTGTTTTGGGTACAGGAGGTCCCGagaagtttccactgcaatcgtacgtgtactgtggcaacacagctataacagacagtttcacgccaaccgC DS129582 GGGTGAGTAGTCTAGTGGTATGATTCCTGTTTTGGGTACAGGAGGTCCCGagaagcttccactgcaatcgtacgtgtactgtggcaacacagctataacagacagtttcacgccaaccgo

\section{Togen-1_DR}

\section{3' boundaries}
ZV7 NA1020 $[50544-51093)$
ZV7 NA21 [1 $[139424-138755]$
Zv7-scaff fold2628_[61683-62495
chr11_[37646849-37647135]
chr12_[32869914-32869431]
chr13_[11700615-11699786
chr14_[28318614-28322031]
chr14_[6426330-6426365] 
chr16_[14874220-14877639] chr16 - (18111641-18114787) chr17_[12754170-12753884 chr17- $14203270-1420294$ chr2_[30234513-30234751] chr20-[26127395-26129919] chr20-[477783112-47783911
chr20-[47970334-47970590 chr20_[7210638-7210074] chr21_[41165320-41165079 chr22_[24295736-24295160 chr24-[39351951-3935248] chr3_[881150-881350] chr4_[31692477-31692425] chr4_[39729528-39728635 chr 7 [24116604-241177775 chr7_[53451622-53451872] chr8 [5633558-5631681] chr $[$ [42228309-42228545]

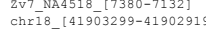

\section{5' boundaries}

\section{chr14_[32752097-32755510} chr17_[2359635-2357363]
chr18 [48922789-48923 chr20-[43152697-43150437]
chr7 [24109574-24110015]

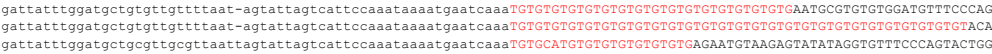

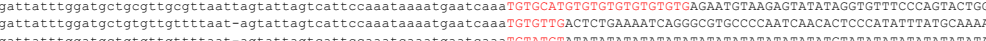
gattatt ggatgctgtgttgttttaat-agtattagtcattccaaatcaaatgaatcaaaTGTATGTATATATATATATATATATATATATATATATATGTATATATATATATATATA

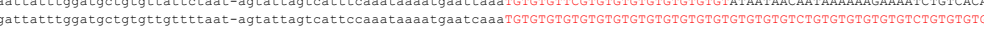
gattatt tggatgctgtgttgttttaat-agtattagtcattccaaataaaa tgaatcaaa TGTGTGTGTATTCAAAATTTTGATCTCCAAATATACAGCTTAATGCACAAATGTTTTM

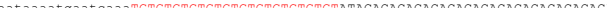
gattat ttggatgctgtgttgttttaat-agtattagtcattccaaataaaa tgaatcaaa TGTGTGTGTGTGTGTGTGTGTGTGTGTGTGTCGTAGCAATGGAGCCGCTGGTTCGCTG ggttatatggatgctgtgttgctttaattagtattagtcattccaataaaattaatcaaaTGTGTGTGGATGTITCCCAGAGATGGGTTGTGGCTGGAAGGGCATCTGCTGCGTAAAM

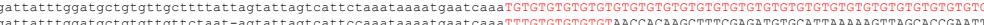

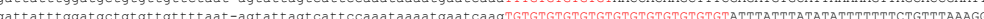
gattatttggatgctgtgttgttctaat-agtattagtcattccaaataaaatgaatcaaa TGTGAGTAAGTCTITTAGCCCGTTTCCACTGAGTGGTGCGATATTGTGTGGTTCGGT gattatt ggatgctgtgttgttttaat-agtattagtcattccaataaaatgatcaaaTGTGTGTGTGTGTATGTATGTATGTATGTATGTATG TGTGTATGTGTGTGTGTGTGT gattatt tggatgctgtgttgttttaat-agtattagtcattccaaataaaatgaatcaaa TGTGTGTGAGAAAGCATGTTTGTGTTTATGAGAGAGCATGTGTGTGTGAGTGAGAGAM

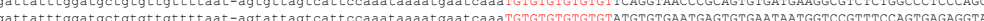

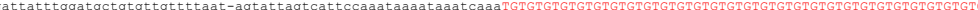
gattatttggttgctgtgttgttctaat-agtattagtcactccaataaatgatcaaaTGTGTGTGTGTGTGTGTGTGTGTGTATGTGTGTGTGTGTGTGTGTGTGTGTGTCCTGC gattatttggatgctgtgttgttttaat-agtattagtcattccaaataaaatgaatcaaaTGTGTGTGTGTG TAAGTCTGTAGAGTTCAACAGGTGTCCTTGACAGGGAACAGGAATT

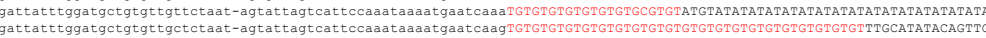
gattatttgaatgctgtgttgctttaattagtattagtcattccaaataaattaatcaaaTGTGTGTGTGTGTGTGTGTGTGTGTGTGTGTGTGTGTGTGTGAATTAAAAAAAAACAT

GCTCAAAGACAAACACTAGTGCTGTTACTGTGTGTGTGTGTGTGTGTATGTGTGTGGATGcgcatgcgttgggagtaacatggcgattgctcccacgcgcaaacatgctgcactaaacaa

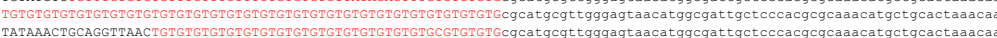
TCCTCCGAGGAGGAGGGTAATGTGTGTGTGTGTGTGTGTGTGTGTGTGTGTGTGTGTGTGcgcatgcgttgggagtaacatggcgattgctcccacgcgcaaacatgctgcactaagca 